\title{
Actividad física adaptada online durante el confinamiento
}

\section{Online physical activity during lockdown}

\author{
D. Quirós González
}

\section{Resumen}

En el momento en el que se decretó el estado de alarma (14 de marzo de 2020), se consideró fundamental que el alumnado del Centro de Recursos Educativos de la ONCE en Alicante continuara realizando actividad física y siguieran manteniendo el contacto entre ellos. Por este motivo, surgió la idea de realizar clases deportivas adaptadas por medio de videollamadas, a través de la aplicación Zoom, todos los sábados, durante alrededor de una hora. La primera semana se conectaron unos 10 niños y niñas de la provincia de Alicante y la siguiente se amplió a todo nuestro ámbito de intervención (Comunidad Valenciana y Región de Murcia). Como resultado del éxito que tuvo esta actividad, se decidió promocionarla a todos los jóvenes del país. Se han sumado más de 40 alumnos en alguna de estas actividades, y en el grupo de WhatsApp que se creó para organizar esta actividad había más de 80 participantes. Además de alumnos, muchos familiares de los participantes y otros profesionales de la oncE también se animaron a participar en esta iniciativa. En las últimas cinco sesiones se añadió un pequeño espacio al término de cada actividad: diferentes deportistas de élite de la ONCE, como Iván Cano (saltador paralímpico de longitud), Mónica Merenciano (judoca paralímpica), Pablo Cantero (jugador de la selección española de fútbol para ciegos) o Esther Ávila (capitana de la selección española de goalball), compartieron con los más pequeños sus experiencias y logros deportivos. Además, contestaron amablemente a las preguntas que les hicieron y les animaron a practicar cada uno de sus deportes una vez terminase esa situación de confinamiento.

\section{Palabras clave}

Deporte adaptado. Actividad física. Discapacidad visual. Confinamiento. Autonomía. Desarrollo psicomotor. 


\begin{abstract}
One of the features of the support afforded students by ONCE's Alicante-based Educational Resource Centre, deemed essential when the state of emergency was called (14 March 2020), was their ongoing participation in physical activity. That informed the initiative to hold hour-long, Zoom-compatible physical education classes for videoconference delivery every Saturday. Ten children in the province of Alicante connected the first week. The following week, reach was extended to include the centre's entire jurisdiction (regions of Valencia and Murcia). In the wake of the success of the activity, participation was subsequently furthered among all youngsters nationwide. Over 40 students took part in some of the activities and over 80 participants signed onto the WhatsApp group created to organise the sessions. In addition to the students themselves, many of their families and other ONCE professionals participated. A highlight added at the end of the last five sessions proved particularly popular. ONCE sport stars shared their experience and achievements in sport with the children. Elites such as Ivan Cano (Olympian long jumper), Monica Merenciano (Paralympian judoka), Pablo Cantero (member of Spain's national football team for the blind) and Esther Ávila (captain of Spain's national goalball team) answered the students' questions in detail and encouraged them to practise sport as soon as lockdown was lifted.
\end{abstract}

\title{
Key words
}

Adapted sport. Physical activity. Visual disability. Lockdown. Independence. Psychomotor development.

\section{Introducción}

Desde hace más de 20 años, los fines de semana del curso escolar, nuestro centro, el CRE (Centro de Recursos Educativos) de la ONCE en Alicante, se encarga de la realización de todo tipo de actividades deportivas: ciclismo en tándem, senderismo, multideporte, fútbol, natación, goalball... El 14 de marzo de 2020, con la entrada en vigor del estado de alarma debido a la pandemia mundial de covid-19, nos vimos obligados a cancelar todas las actividades lúdico-deportivas programadas para ese curso escolar. Por ello, en los días siguientes, se tomó la iniciativa de que los alumnos realizasen algún tipo de actividad física durante el periodo que iban a pasar en sus domicilios.

A día de hoy, podemos encontrar en internet infinidad de recursos multimedia para hacer deporte desde casa, pero ninguno de ellos está adaptado a las características

Quirós, D. (2021). Actividad física adaptada online durante el confinamiento. RED Visual: Revista Especializada en Discapacidad Visual, 77, 305-310. https://doi.org/10.53094/UZMZ9573. 
que tienen nuestros afiliados. Es ahí cuando nació la idea de intentar juntar a nuestros alumnos y realizar, de manera simultánea, una actividad física adaptada por medio de una videollamada grupal. En estas sesiones los diferentes ejercicios estaban diseñados para que una persona con baja visión o sin resto visual pudiera realizarlos de manera autónoma. Únicamente necesitaban un pequeño espacio de su domicilio y un teléfono móvil con conexión a internet y cámara. Esta actividad se realizaba en directo con el objetivo de motivar a los jóvenes y poder corregirles si realizaban algún ejercicio de manera errónea.

\section{Objetivos}

El objetivo principal de esta actividad era que los alumnos se divirtieran durante el confinamiento practicando actividad física desde sus casas. Del mismo modo, concienciarles a ellos y a sus familias de llevar un estilo de vida saludable por medio de la práctica de ejercicio físico y una buena alimentación. En estas sesiones, además, se buscaban otros objetivos no menos importantes. La socialización y la convivencia (no física, pero sí emocional) entre nuestros pequeños afiliados era algo inherente durante la realización de esta actividad.

\section{Destinatarios y temporalización de la actividad}

Cuando se planteó realizar esta actividad, los destinatarios iban a ser únicamente los alumnos del CRE de la ONCE en Alicante con edades comprendidas entre 5 y 15 años. Se creó un grupo de WhatsApp en el que se informaba a las familias de cada una de las sesiones que se iban a realizar. También se utilizaron las redes sociales de nuestro centro para promocionar e invitar a más participantes. Con ello, se consiguió que personas de otros puntos de la Comunidad Valenciana decidieran unirse a esta iniciativa. Pero el punto álgido se alcanzó cuando jóvenes de distintos lugares de nuestro país se animaron también a participar en estas sesiones deportivas adaptadas.

En la primera sesión se conectaron, por medio de la aplicación Zoom, 15 participantes. Con el paso de las sesiones, el número fue aumentando hasta llegar a tener alrededor 30 en cada una de ellas. Además de los alumnos, familiares de estos realizaban la actividad con nosotros por varios motivos: para llevar una vida más saludable y también para ayudar a sus hijos o hermanos en la realización de los ejercicios más 
complicados. Por último, comentar que un gran número de profesionales de la ONCE (directores de centro, trabajadores sociales, maestros, instructores de tiflotecnología, etc.) también se animaron a participar en las sesiones. Debido a este alto número de participantes, se decidió crear un grupo de WhatsApp en el que estuvieran todas aquellas personas interesadas. El número total llegó a superar los 80 integrantes.

Esta actividad, que se realizó durante el periodo de confinamiento, tuvo las siguientes fechas de las sesiones y número de participantes:

Tabla 1. Participantes totales sin contar a los familiares de cada uno de los alumnos que realizaron esta actividad

\begin{tabular}{|c|c|}
\hline Fecha de la sesión & N.o de participantes \\
\hline $11-04-2020$ & 15 \\
\hline $18-04-2020$ & 12 \\
\hline $25-04-2020$ & 24 \\
\hline $02-05-2020$ & 28 \\
\hline $09-05-2020$ & 29 \\
\hline $16-05-2020$ & 26 \\
\hline $23-05-2020$ & 24 \\
\hline $30-05-2020$ & 21 \\
\hline $06-06-2020$ & 24 \\
\hline $13-06-2020$ & 23 \\
\hline $20-06-2020$ & 18 \\
\hline
\end{tabular}

\section{Metodología}

Se utilizó una metodología de mando directo en la que el profesor de educación física del CRE de la ONCE en Alicante planteaba, por medio de una videollamada grupal, diferentes ejercicios adaptados para personas con discapacidad visual y que fueran realizables dentro de un hogar. Se buscaron ejercicios motivantes, divertidos y, como se ha comentado con anterioridad, adaptados a las características de este grupo de población. Al término de estas sesiones, se contaba con un total de aproximadamente 40 ejercicios distintos, los cuales los deportistas realizaban de manera autónoma, obje- 
tivo que se buscaba desde la primera sesión para que una vez terminase esta iniciativa pudieran seguir haciendo deporte con total autonomía y sin necesidad de apoyo.

Figura 1

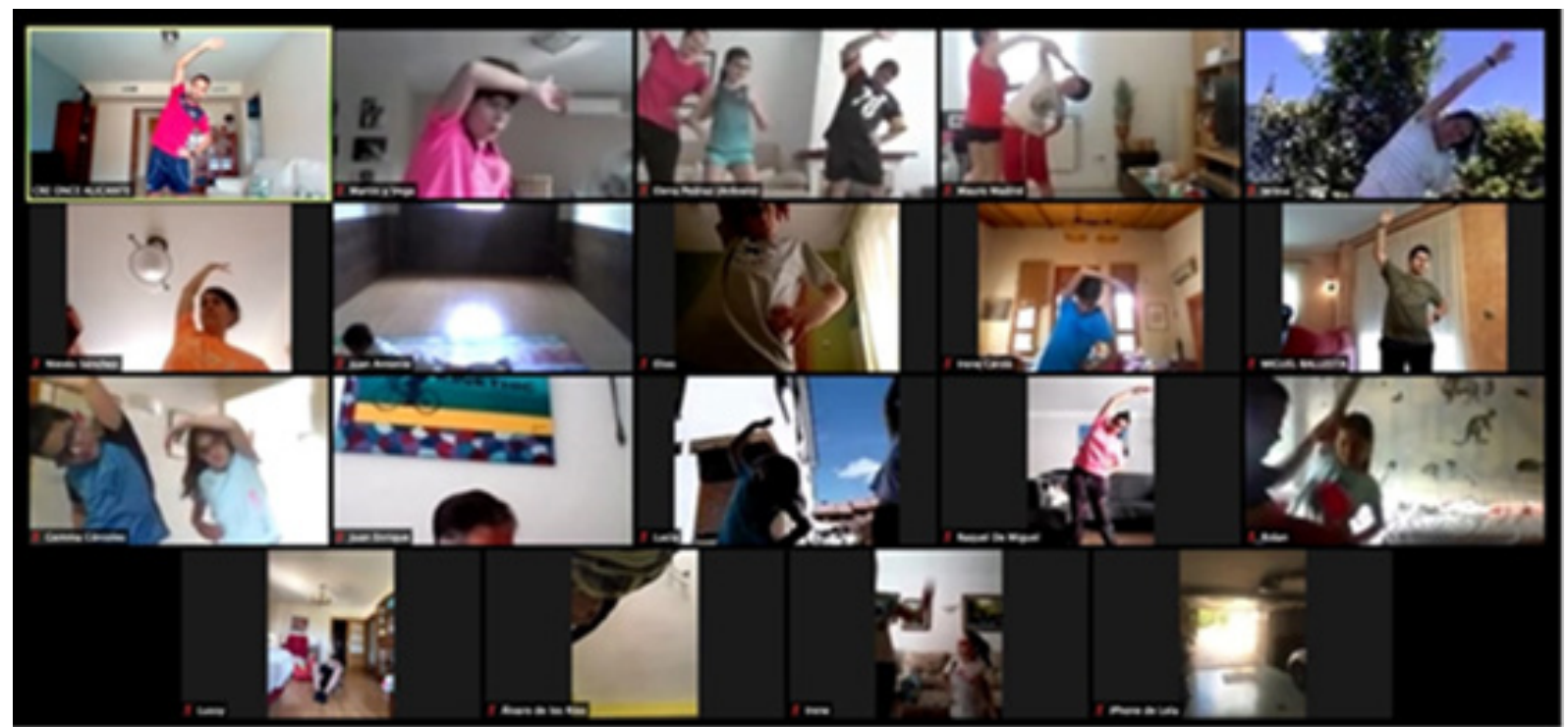

Antes de la realización de las sesiones se diseñaban cada una de estas en función de las características de los participantes: la franja media de edad de estos estaba entre los 5 y los 13 años. Por este motivo, tenían que planificarse actividades sencillas, motivantes, divertidas y, sobre todo, adaptadas a la discapacidad visual. Antes de cada ejercicio se realizaba una explicación minuciosa de cada tarea (atendiendo a los distintos grupos musculares que tomaban parte) para que los participantes pudiesen realizar dichos ejercicios de manera correcta y, sobre todo, autónoma.

Por otro lado, teniendo en cuenta que el mundo del deporte es algo muy presente en nuestro día a día (en la televisión la mayoría de deportistas se convierten en ídolos de los más pequeños, quienes les imitan tratando de llegar algún día a ser como ellos), se añadió un espacio al término de las últimas cinco sesiones en las que diferentes deportistas paralímpicos de nuestro país les contaron a los participantes cómo entrenaban durante el confinamiento, qué logros deportivos habían conseguido y cómo compatibilizaban el deporte con su vida académica, profesional y familiar. Además, se eligieron deportistas de diferentes disciplinas, como goalball, ciclismo en tándem, atletismo, fútbol y judo. El objetivo final era que, una vez se terminase el confinamiento, se animaran a practicar alguno de estos deportes. 


\section{Resultados y conclusiones}

Se puede cuantificar el número de participantes totales y distintos para conseguir una cifra de resultados y compararlo con otras actividades realizadas, pero el objetivo de esta actividad no era ese. Desde la primera sesión, se buscaba que los integrantes del grupo disfrutaran haciendo deporte y se olvidasen, durante esa hora que duraba la actividad, de todas las noticias que escuchábamos por aquellos días acerca de la covid-19.

Muchas familias, al término de esta iniciativa, nos comentaron que, gracias a esta, sus hijos habían hecho deporte y la cuarentena se les había pasado más rápido. Además, habían hecho nuevas amistades, tanto entre niños como padres, que, a día de hoy, se mantienen en contacto y organizan distintas actividades. Aun no perteneciendo todos al mismo centro de la ONCE, los participantes se sentían parte de un gran grupo que buscaba disfrutar y socializar por medio de la práctica deportiva.

En definitiva, ha sido un verdadero placer el organizar y disfrutar de una actividad de estas características, y sentir el cariño y la acogida que esta tuvo durante y después de su realización.

Diego Quirós González. Maestro y Especialista de Educación Física. Centro de Recursos Educativos de la ONCE en Alicante. Avenida de Denia, 171; 03559 Alicante (España). Correo electrónico: dqg@once.es. 